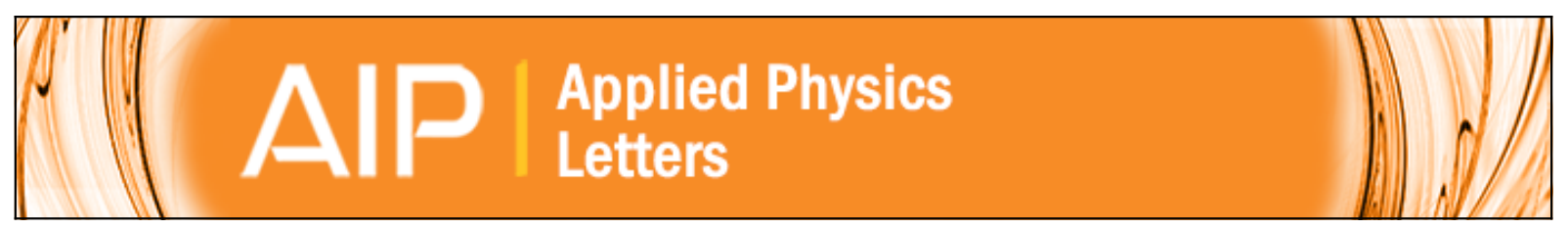

Effect of proton bombardment on InAs dots and wetting layer in laser structures

I. O'Driscoll, P. Blood, P. M. Smowton, A. Sobiesierski, and R. Gwilliam

Citation: Applied Physics Letters 100, 261105 (2012); doi: 10.1063/1.4730964

View online: http://dx.doi.org/10.1063/1.4730964

View Table of Contents: http://scitation.aip.org/content/aip/journal/apl/100/26?ver=pdfcov

Published by the AIP Publishing

AlP

Create a profile.

Sign up today! 


\title{
Effect of proton bombardment on InAs dots and wetting layer in laser structures
}

\author{
I. O'Driscoll, ${ }^{1}{ }^{1, a)}$ P. Blood, ${ }^{1}$ P. M. Smowton, ${ }^{1}$ A. Sobiesierski, ${ }^{1}$ and R. Gwilliam ${ }^{2}$ \\ ${ }^{1}$ School of Physics and Astronomy, Cardiff University, Queens Buildings, The Parade, Cardiff CF24 3AA, \\ United Kingdom \\ ${ }^{2}$ Advanced Technology Institute, FEPS, University of Surrey, Guildford, Surrey GU2 7XH, United Kingdom
}

(Received 24 April 2012; accepted 9 June 2012; published online 26 June 2012)

\begin{abstract}
The effect of proton bombardment on carrier lifetime and photoluminescence of InAs quantum dots was measured. Optical absorption and transmission electron microscopy show the dots retain their integrity under bombardment. A decrease in ground state photoluminescence with increasing dose is not explained by the decrease in dot carrier lifetime alone, but also by bombardment-induced non-radiative recombination in the wetting layer, which reduces the dot electron population at fixed excitation. To exploit the relative radiation immunity of quantum dots, it is necessary to maximise the dot density and capture probability per dot to minimize the effect of wetting layer recombination. (C) 2012 American Institute of Physics. [http://dx.doi.org/10.1063/1.4730964]
\end{abstract}

The conversion of beamed optical power from a laser into electrical energy is an attractive proposition for many applications. One of these includes the potential to capture solar energy in space and deliver it continuously, on demand, to anywhere on earth. ${ }^{1}$ To operate in space, lasers must meet stringent radiation hardness standards. ${ }^{2}$ Among the advantages claimed for quantum dots in optoelectronic devices such as lasers is their relative immunity to particle and radiation bombardment where non-radiative processes are known to result in the degradation of traditional quantum well devices. These claims rest on the reduction in the area of material exposed to the radiation, ${ }^{3}$ though there may be benefits from localisation of carriers within each dot. ${ }^{4,5}$ Here we quantify the effect of proton bombardment in quantum dot material and discuss implications for laser design to withstand such harsh environments using fundamental measurements including modal optical absorption, transmission electron microscopy (TEM), photoluminescence (PL), and PL lifetime as a function of proton dose. It is observed that the nonradiative recombination rate saturates for increasing proton dose and we suggest that multiple atomic displacements agglomerate in a dot to form a single recombination center. We find a decrease in luminescence at the ground state (GS) of InAs dots which is not explained simply by the reduction in the measured carrier lifetime in the dots. We propose that defects produced by bombardment in the wetting layer (WL) have a significant influence on the light output of the dot states at a fixed excitation rate into the WL. Robust laser designs should therefore maximise the dot density and, if possible, the capture rate per dot to minimise the effect of WL recombination.

The structure has five layers of InAs quantum dots grown in an $\mathrm{In}_{0.15} \mathrm{Ga}_{0.85}$ As well in a GaAs core and an $\mathrm{Al}_{0.45} \mathrm{Ga}_{0.55} \mathrm{As}$ cladding waveguide. Samples were bombarded in a direction misaligned from the surface normal at an energy of $230 \mathrm{keV}$, chosen to maximize atomic displacements at the depth of the dot layers calculated using

a) odriscollip@ cardiff.ac.uk.
SRIM2011, ${ }^{6}$ with doses between $3 \times 10^{12}$ to $1 \times 10^{14}$ protons $\mathrm{cm}^{-2}$. We estimate a dose of $3 \times 10^{12}$ protons $\mathrm{cm}^{-2}$ corresponds to an exposure time in space of approximately 30 years. ${ }^{7}$ From the simulations, the atomic displacement rate at the dot layers is $5 \times 10^{-4}$ per surface proton dose per $\AA$ so taking a dot to be $30 \AA$ high and cross sectional area $12 \mathrm{~nm} \times 12 \mathrm{~nm}$, the number of displacements per unit area in a layer $30 \AA$ thick is $1.5 \times 10^{-2} \times$ surface proton dose. The number of displacements produced in a single dot of area $1.4410^{-12} \mathrm{~cm}^{2}$ by a dose $d$ protons per unit area is $\left[2.2 \times 10^{-14} \times d\right]$, which we write below as $[\alpha d](\alpha$ has dimensions of area). On average $1 \times 10^{14}$ protons $\mathrm{cm}^{-2}$ produces two displacements per dot. If each of these displacements produces a defect state which generates non-radiative recombination, the irradiation-induced recombination rate per unit area in a layer of $N_{d}$ dots $\mathrm{cm}^{-2}$ is $R_{n r}(d)$ $=\left(2.2 \times 10^{-14}\right) N_{d} d / \tau_{n r}$, where $\tau_{\mathrm{nr}}$ is the non-radiative lifetime at each defect. Although the proton doses here are similar to those used in Ref. 8, their proton energy was much higher at $2.4 \mathrm{MeV}$ so the displacement rate in our experiments is probably much higher because we have chosen the proton energy to maximize the atomic displacement rate in the dot layer itself.

Modal optical absorption measurements on a sample bombarded with $1 \times 10^{12}$ protons $\mathrm{cm}^{-2}$ show little change in the spectrum compared with un-bombarded material, as shown in Fig. 1, therefore, using the Einstein relations, we do not expect the radiative lifetime to change with bombardment. TEM images on a similar sample subject to a dose of $1 \times 10^{14}$ protons $\mathrm{cm}^{-2}$ also show that the physical form of the dots remains unchanged. We conclude that these doses do not destroy the integrity of the dots by intermixing.

The PL was pumped with an excitation wavelength of $780 \mathrm{~nm}$ and carriers were supplied to the dots via the WL. The spectra, measured at $300 \mathrm{~K}$ and $100 \mathrm{~K}$ using an Ando AQ-6315B spectrum analyzer, show peaks at approximately $880 \mathrm{~nm}, 1200 \mathrm{~nm}$, and $1275 \mathrm{~nm}$ due to the WL, quantum dot excited state, and GS transitions at $300 \mathrm{~K}$, respectively. We measured the total carrier lifetime at the GS peak using a 


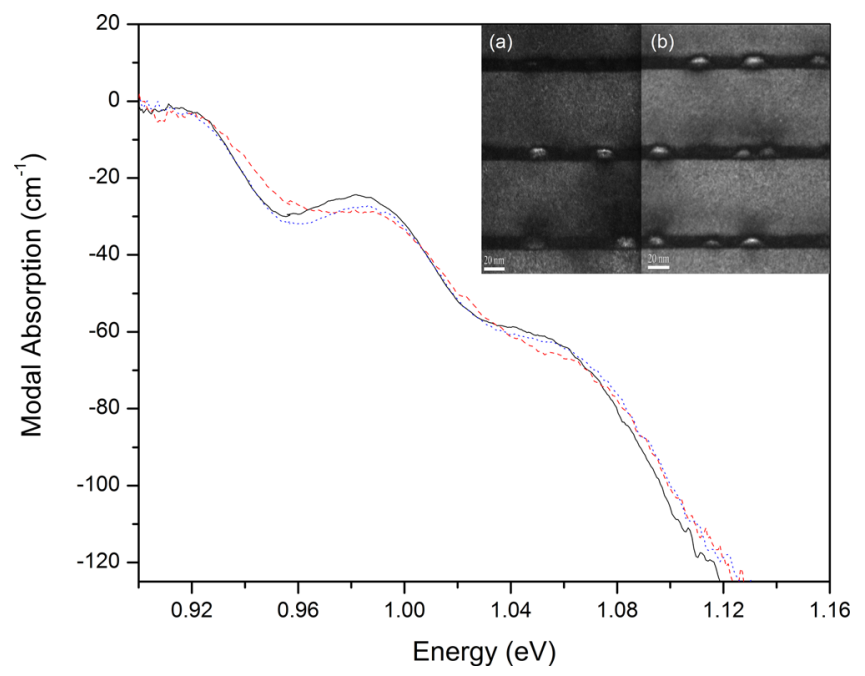

FIG. 1. Modal absorption spectra for the unbombarded sample (solid line) and bombarded samples using a proton dose of $1 \times 10^{11}$ protons $\mathrm{cm}^{-2}$ (dotted line) and $1 \times 10^{12}$ protons $\mathrm{cm}^{-2}$ (dashed line) at $300 \mathrm{~K}$. Inset: TEM images of the quantum dot layers courtesy of Integrity Scientific Ltd. (a): Unbombarded sample. (b): Proton dose of $1 \times 10^{14}$ protons $\mathrm{cm}^{-2}$.

streak camera with a resolution of 3 ps at a sample temperature of $100 \mathrm{~K}$, where the measurement was more sensitive to bombardment-induced changes.

The lifetime is reduced from 140 ps in the control sample to about $30 \mathrm{ps}$ at a dose of $1 \times 10^{14}$ protons $\mathrm{cm}^{-2}$. Data for the reciprocal measured lifetime $\left(\tau_{\text {meas }}\right)$ at the GS for a bandwidth of $50 \mathrm{~nm}$ is shown in Fig. 2; this is the total recombination rate $\left(R_{t o t}\right)$ which is the sum of the radiative and non-radiative rates in the unbombarded material plus the non-radiative rate due to irradiation-induced defects, $R_{n r}(d)$. At low dose, where the probability of atomic displacement in a dot, or within the extent of its wavefunction, is less than one, the number of displacements seen by the dots should increase linearly with dose (as shown above), which is consistent with our observations. At higher doses, multiple displacements are created at each dot and if each of these produces an independent recombination centre, the nonradiative rate should continue to increase with dose. How-

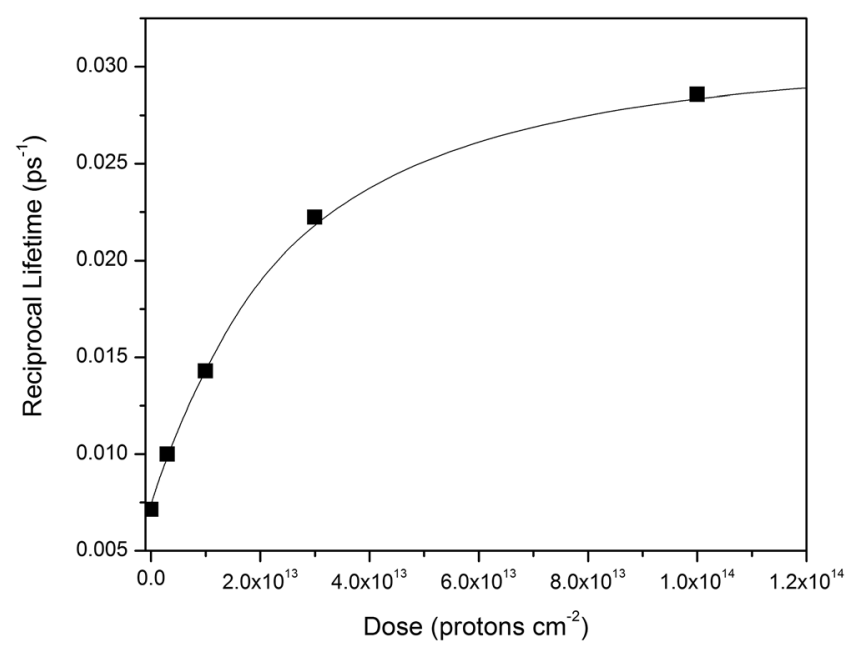

FIG. 2. Reciprocal of the experimentally measured GS carrier lifetime (black squares) and modeled carrier lifetime (black line) versus proton dose at $100 \mathrm{~K}$. ever, the data in Fig. 2 shows that non-radiative rate saturates at high dose and we suggest this is because the displacements at a single dot agglomerate into a single defect so the number of defects does not exceed one per dot.

If we adopt this simple model in which the non-radiative rate is determined by the production of defects at undamaged dots, then as irradiation proceeds with time the increase in the number of dots with a recombination centre produced by an increment of $\Delta d$ in dose is

$$
\Delta N_{d}^{n r}(d)=\left\{N_{d}-N_{d}^{n r}(d)\right\} \alpha \Delta d,
$$

so the measured carrier lifetime of the ensemble of $N_{d}$ dots per unit area should vary with dose as

$$
\frac{1}{\tau_{\text {meas }}}=\frac{1}{\tau_{0}}+\frac{1-\exp (-\alpha d)}{\tau_{n r}}
$$

where $\tau_{0}$ is the lifetime in the unbombarded sample and $\alpha d$ is the displacement production per dot. Using a displacement production of $\left.\left[4 \times 10^{-14} \times \mathrm{d}\right)\right]$ and a non-radiative lifetime of $45 \mathrm{ps}$ a fit of the whole range of experimental data was obtained as shown in Fig. 2. This is of similar magnitude to our initial estimate of $\left[2.2 \times 10^{-14} \times \mathrm{d}\right]$ and is within the uncertainty in the dot area and height; recombination in the dots may be sensitive to deep states produced in their vicinity.

We next examine the GS PL data. At fixed excitation into the dots themselves the ground state PL efficiency is the ratio of the radiative rate per dot and total recombination rate per dot which is proportional to the reciprocal of the measured lifetime, $\tau_{\text {meas }}$. Thus the efficiency is $\left(R_{\text {rad }} \tau_{\text {meas }}\right)$. If the radiative lifetime per dot is not changed by bombardment because there are no structural changes, the PL should be proportional to $\tau_{\text {meas }}$ : as the overall lifetime gets shorter due to increased non radiative recombination so the PL is

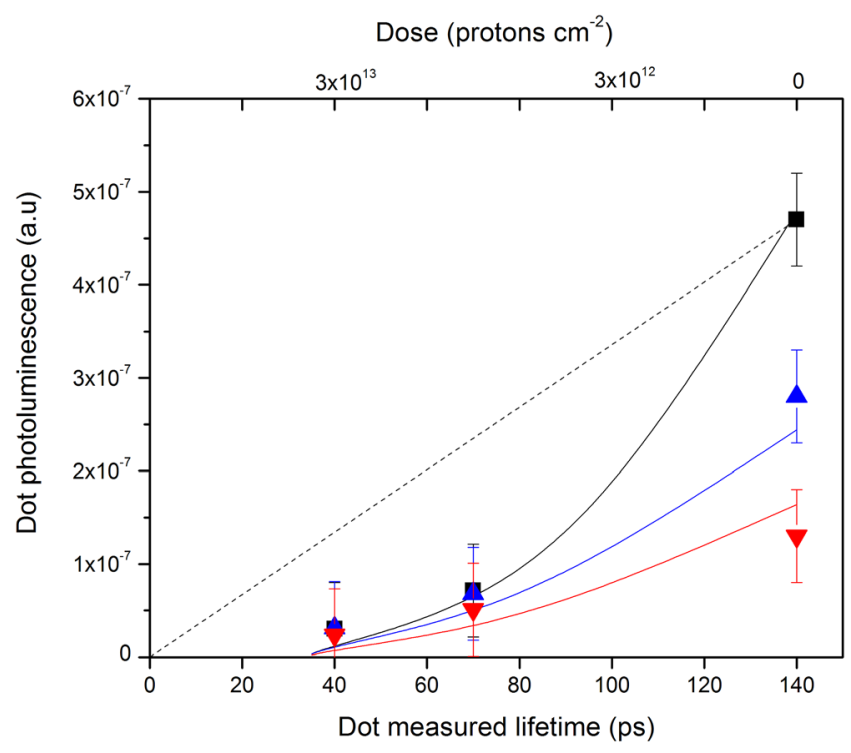

FIG. 3. Experimentally measured GS PL versus measured ground state lifetime (bottom X-axis) and proton dose (top x-axis) for pump powers of 36 $\mathrm{mW}$ (squares), $18 \mathrm{~mW}$ (triangles), and $12 \mathrm{~mW}$ (inverted triangles) at $100 \mathrm{~K}$. The solid lines are fits including WL recombination for the same pump power. The dash line indicates the linear behaviour expected for a fixed dot carrier population. 
reduced. This simple proportionality between the PL and $\tau_{\text {meas }}$ is not observed in the data at $100 \mathrm{~K}$ shown in Fig. 3. Starting with un-bombarded material with a lifetime of 140 ps the initial decrease of PL with decreasing lifetime (increasing dose) is much faster than a linear behavior. Similar behavior is observed at $300 \mathrm{~K}$. It has been suggested that defects may enhance capture into the dots ${ }^{4}$ in which case we would expect an initial linear decrease in PL with increasing dose then for the PL to be enhanced above the dash line due to improved capture. Competition between the increased non-radiative recombination in the dots and the capture onto the dots does not account for this behavior.

Since the excitation for PL measurements generates carriers in the WL (similar to injection from a $\mathrm{p}-\mathrm{n}$ junction), we suggest that recombination at bombardment-induced deep states in the WL competes with capture of carriers onto the dots, reducing the carrier population in the WL and hence on the dots as the proton dose increases. This hypothesis is supported by the observed reduction in PL from the WL as a function of dose shown in Fig. 4. Taking a WL thickness of $50 \AA$, the displacement rate per unit area in the WL is $\left(5 \times 10^{-4} \times 50 \times \mathrm{d}\right)$ which we represent as $\gamma_{w} d$ with $\gamma_{w}=2.5 \times 10^{-2}$. Solving the following set of rate equations in the WL and the dots in the steady state, we have calculated the carrier population and PL of the dots and WL as a function of dose. If the electron-hole pair generation rate per unit area in the WL (proportional to the incident photon flux) is $G$, the steady state condition for the number of carriers in the WL is given by

$$
G=\left[\frac{n_{w}}{\tau_{0, w}}+n_{w} C_{w} \gamma_{w} d\right]+C_{d} n_{w}\left(N_{d}-n_{d}\right),
$$

where $n_{w}$ is the number of carriers in the WL per unit area, $\tau_{0, w}$ is the measured lifetime in the WL prior to bombardment, $C_{w}$ and $C_{d}$ are the capture coefficients for electrons from the WL to the deep states in the WL and to the dots respectively $\left(\mathrm{cm}^{2} \mathrm{~s}^{-1}\right), N_{d}$ is the number of dots $\left(\mathrm{cm}^{-2}\right), n_{d}$ is the number of carriers on the dots $\left(\mathrm{cm}^{-2}\right)$. The term in [] is

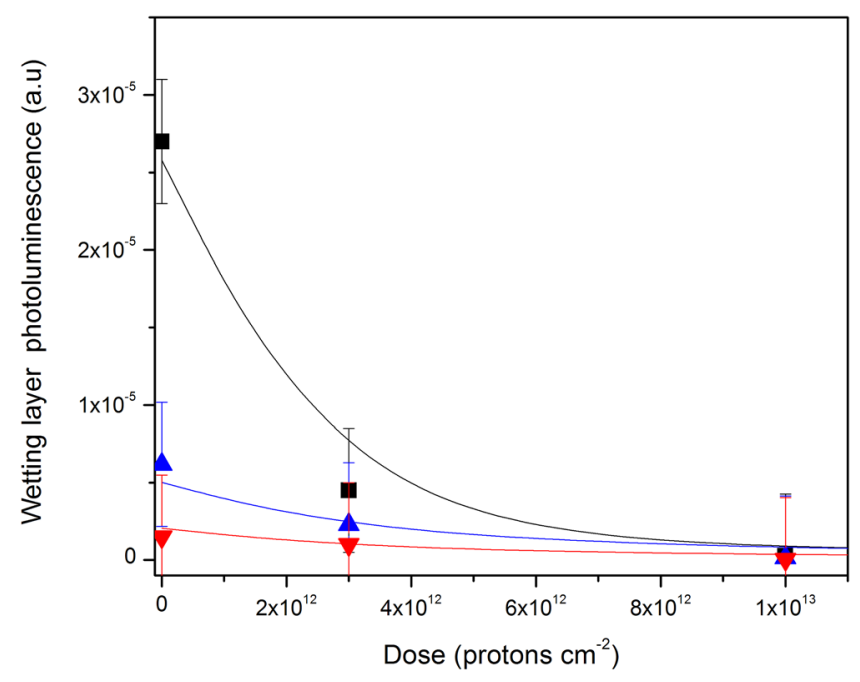

FIG. 4. Experimentally measured WL PL versus dose for pump powers of $36 \mathrm{~mW}$ (squares), $18 \mathrm{~mW}$ (triangles), and $12 \mathrm{~mW}$ (inverted triangles) at $100 \mathrm{~K}$. The solid lines are fits to the experimental data with the same parameter values as in Fig. 3. the total recombination rate in the WL which we write as $\left(\mathrm{n}_{\mathrm{w}} / \tau_{\mathrm{w}}\right)$.

The steady state condition for the dots is given by

$$
C_{d} n_{w}\left(N_{d}-n_{d}\right)=\frac{n_{d}}{\tau_{\text {meas }}},
$$

where $\tau_{\text {meas }}$ is the measured carrier lifetime in the dots. Combining these equations gives a quadratic which we can solve for the number of carriers on the dots $n_{d}$,

$$
n_{d}^{2}-\left(G \tau_{\text {meas }}+\frac{1}{C_{d} \tau_{w}}+N_{d}\right) n_{d}+G \tau_{\text {meas }} N_{d}=0 .
$$

We calculated $n_{d}$ as a function of dose through the terms $1 / \tau_{\mathrm{w}}$ and $\tau_{\text {meas }}$ measured as a function of dose using an estimated value for $\gamma_{w}$ and the nominal dot density. The plot of dot PL versus measured lifetime in Fig. 3 was obtained as follows. The PL for the dots is $\left(n_{d} / \tau_{\text {rad }}\right)$ (with $\tau_{\text {rad }}=1 \mathrm{~ns}$ obtained from the measured absorption cross section ${ }^{9}$ ) and was calculated by making sensible adjustment to $C_{w}$ and $C_{d}$ to match the experimental data in Fig. 3, and changing $G$ proportional to the incident power for each of the curves. The same scaling factor to arbitrary PL units was used throughout. The WL PL was calculated as proportional $n^{2}{ }_{w l}$ and was scaled to the measured PL as a function of dose in Fig. 4, using the same parameters as for the fits in Fig. 3. The measured lifetime in the WL in the unbombarded sample was $60 \mathrm{ps}$. The value used for $C_{w}$ corresponds to a deep state capture cross section of $1 \times 10^{-14} \mathrm{~cm}^{2}$ and we used $C_{d}=0.07 \mathrm{~cm}^{2} \mathrm{~s}^{-1}$. Figs. 3 and 4 show this model provides a good description of the variation of WL and dot PL with proton bombardment for three different excitation rates into the WL with the same set of parameters. This suggests that the rapid decrease in dot PL with carrier lifetime due to proton bombardment in Fig. 3, at fixed excitation into the WL, is due to competition for carriers by recombination at radiationinduced defects in the WL. This should not be surprising given that the dots account for only a fraction of the area of the WL. LEDs and laser diodes operate by electrical injection into the WL and the same model should apply.

From our fits to the experimental data, we estimate that for a dose of $3 \times 10^{12}$ protons $\mathrm{cm}^{-2}$ the current for a fixed optical gain is increased by a factor of about four, with the WL accounting for $80 \%$ of the total recombination current.

We conclude that the number of deep states saturates with increasing proton dose suggesting that multiple atomic displacements agglomerate at a dot to form a single recombination center. Furthermore, the increase in the non-radiative recombination rate in the WL due to proton damage accounts for the fact that the PL from the GS decreases with dose much faster than due to the reduction in the measured GS carrier lifetime alone. Bombardment-induced non-radiative recombination in the WL can cause a greater reduction in GS PL output than the deep states produced in the dots themselves. Our experiments have been taken to higher damage production rates than some former studies, and our results suggest that for prolonged exposure to radiation, defects in the WL have a significant influence on the performance. Therefore to exploit the benefits of quantum dots for lasers used in space and other harsh environments, it is necessary to minimize the 
deleterious effect of defects in the WL by maximizing the dot density and if possible the capture rate per dot.

${ }^{1}$ Department of Defense, Space-Based Solar Power as an Opportunity for Strategic Security (National Security Space Office, 2007).

${ }^{2}$ European Cooperation for Space Standardization, ECSS Secretariat, ESAESTEC Requirements \& Standards Division, Noordwijk, The Netherlands, "Space Engineering-Method for the calculation of radiation received and its effects and a policy for design margins," ECSS-E-ST-10-12C (2008), http://www.ecss.nl/forums/ecss/dispatch.cgi/standards/showFile/100701/ d20081115091445/No/ECSS-E-ST-10-12C(15November2008).pdf.

${ }^{3}$ R. Leon, G. M. Swift, B. Magness, W. A. Taylor, Y. S. Tanga, K. L. Wang, P. Dowd, and Y. H. Zhang, Appl. Phys. Lett. 76(15), 2074-2076 (2000).
${ }^{4}$ S. Marcinkevičius, J. Siegert, R. Leon, B. Cechavicius, B. Magness, W. Taylor, and C. Lobo, Phys. Rev. B 66, 235314 (2002).

${ }^{5}$ C. Ribbat, R. Sellin, M. Grundmann, D. Bimberg, N. A. Sobolev, and M. C. Carmo, Electron. Lett. 37(3), 174 (2001).

${ }^{6}$ J. F. Ziegler, J. P. Biersack, and U. Littmark, The Stopping and Range of Ions in Solids (Pergamon, New York, 1985).

${ }^{7}$ J. Feynman, G. Spitale, J. Wang, and S. Gabriel, "Interplanetary proton fluence model: JPL 1991,” J. Geophys. Res. 98(A8), 13281-13294, doi:10.1029/92JA02670 (1993).

${ }^{8}$ F. Guffarth, R. Heitz, M. Geller, C. Kapteyn, H. Born, R. Sellin, A. Hoffmann, D. Bimberg, N. A. Sobolev, and M. C. Carmo, Appl. Phys. Lett. 82(12), 1941-1943 (2003).

${ }^{9}$ I. O'Driscoll, P. Blood, and P. M. Smowton, IEEE J. Quantum Electron. 46, 525-532 (2010). 